\title{
Eye-tracking in Second Language Reading
}

Ivana Roncevic, University of Zagreb

\begin{abstract}
In this study eye tracking software was used to analyse the ways in which university students, adult competent speakers of English at C1 level, process information when reading texts in the target language, and which strategies they use in order to summarise a given text. Research results point to four different reading techniques used by the participants in the study: partly selective fast linear, partly selective slow linear, selective structural and nonselective reading strategies. In comparison with previous research with reading tasks in L1, results show that readers take more time when reading in L2. In addition, there is a need for skill enhancement for the purpose of improved text structuring in reading tasks in the English language.
\end{abstract}

Key words: Cluster Analysis, Eye-tracking, EFL, Reading Strategies, Second Language Learning.

\section{Introduction}

The use of eye tracking in psycholinguistic research is relatively novel, and has been successfully applied in the past several decades. Its use in L2 learning and acquisition has been helpful in providing insight into how learners process information when reading or listening to a text. Information on how readers process information in L2 reading comprehension tasks can help to advance the learning process and improve learner reading skills. In order to do so, we first need to identify and analyse current reading strategies.

Previous research in reading comprehension theory, and empirical studies, have shown that reading comprehension includes processing on micro and macro levels, and that macroprocessing has significant influence on the ways a reader will read the text at the micro-level. Readers apply a specific global processing strategy, which affects their subsequent microprocessing methods (see Kintsch and van Dijk, 1978). In spite of this awareness, most theoretical and empirical studies in this field have been focused on micro-processing, and until recently there has been a common assumption that such processes form the basis of all reading comprehension tasks (McKoon and Ratcliff, 1992). Studies from last century (see Graesser et al., 1995) have dealt with the processing of figures of speech (see Garrod and Sanford, 1994) and causal relations in a text. According to Hyöna et al. (2002), the importance of information 
processing at macro-level, which focuses on topic comprehension, has been neglected in favour of micro-processes.

Another limitation of studies completed in the previous century is the accuracy of eye tracking data. Research on reading strategies depends on the data acquired from measuring eye fixations and saccades. In adults, fixations usually last between 100 and 800 milliseconds, while university students typically measure 250 milliseconds. Eye movement from one word to another can last from 10 to 20 milliseconds, and from one line to the next it can measure between 60 and 80 milliseconds. Consequently, when measuring fixations and saccades, it is important to use an eye tracker with high sampling rates in order to achieve high temporal accuracy. The average error of a sampling rate of $1000 \mathrm{~Hz}$ will amount to only 0.5 milliseconds, which is acceptable when measuring fixations and saccades. Most research in the past relied on eye trackers with a sampling rate of $60 \mathrm{~Hz}$. This sampling rate results in an average error of 8 milliseconds, which is acceptable when measuring fixations, but too unreliable for measuring saccades. Nowadays studies applying eye tracking for reading strategies rely on $500 \mathrm{~Hz}$ to 1000 $\mathrm{Hz}$ sampling rates.

In this study we will focus on analysing the macro-processes in reading, that is, on global information processing techniques and strategies applied by university students when presented with a reading comprehension task involving two texts in L2, in this case, English.

Previous research dealing with macro-processes dealt with expository types of texts (Fletcher, 1986; Hyöna et al., 2002; Lorch, R.F. and Lorch, E.P., 1996), as well as narrative texts (Fletcher and Bloom, 1988). The limitations of studies conducted in the past century pertain to applying a deductive approach by modelling information processing strategies of adult readers and applying them to average reading time and average recall. As a result, the studies focused on one generic type of average reader to describe their processing strategy. However, research has shown that language learners and readers in general apply different strategies in reading. Therefore, this study allows for variations in reading strategies used in L2 reading comprehension tasks when dealing with an expository text.

\section{Expository Text Comprehension}

The empirical part of this study deals with information processing in an English language expository text. Such a text usually has a general topic (such as social media) that is correlated with additional topics (for example, communication, social networks, networking, Internet privacy) and is additionally divided into subtopics (fake news in social networks, privacy concerns in social networks, as examples). In the process of text comprehension, the reader aims at understanding the topic(s) of the text, its subtopics, and the connections between them. According to Hyöna et al. (2002), it is challenging for a reader to process the structure of the topics presented in the text, because subtopics are chosen in an arbitrary way, and presented in an arbitrary order, which makes it harder to structure the topic. In addition, expository texts 
usually focus on the subtopics, so that the reader has to establish the connections between the topics on their own. New topics can be introduced abruptly, which results in some difficulty for the reader, who needs to process this information independently. This means that they need to read carefully in order to identify a sentence introducing a new topic and to be able to elaborate the structure of the text. It is thus expected that sentences with new topics will require a longer processing time in comparison to time dedicated to other sentences, as has been proven in previous research (e.g., Hyöna, 1995; Hyöna et al., 2002; Kieras, 1981; Lorch, R.F., et al., 1985, 1987). Studies have also shown that the greater the difference between the topics, the greater the processing time (Lorch, E.P. et al., 1987; Lorch, R.F., et al., 1985, 1987; Vauras et al., 1992). Thus, if there is a shift in both the subtopic (for example, fake news versus privacy in social networks) and a superordinate topic (such as social media versus in-person communication), it will require a longer processing time than a subtopic-only change within the same superordinate topic.

According to Haberlandt and Graesser (1989), topic sentences require longer processing time and more attention because they contain new information, which requires more cognitive effort from the reader. Hyöna et al. (2020) emphasise that topic processing also includes the aspect of strategic processing, but only in cases where the reading task is topic oriented. In such cases a global processing strategy is used. Such a strategy is also likely to be used if the text involves topic boundary cues such as numbers or subtitles (Lorch, R.F., and Chen, 1986). Also, the application of a global processing strategy depends on the individual characteristics of a reader, as readers differ in their evaluation of topic structure relevance for a given text. The so-called topic-shift effect, or a reader's sensitivity to the introduction of a new topic in a sentence, is more frequent in adults with a higher rate of recall than those who scored lower in recall (Lorch, R.F., et al., 1987).

\section{Strategy Differentiation Among Readers: Selective and Non-selective Strategies}

Research has shown that readers apply a mosaic of strategies in order to understand a text globally. A successful reader will search for topic sentences and pay attention to the beginning and end of a paragraph (Hyöna et al., 2002; Pressley and Afflerbach,1995). They will also scan the text for particular words and pieces of information, as well as identify logical connections between sentences. In addition, readers will often backtrack to important sentences in a text where the main idea is expressed (Goldman and Saul, 1990).

Previous research indicates that readers apply selective strategies in text processing depending on text coherence and structure. Topic shifts in a text will require additional cognitive effort and processing time and successful readers will pay attention to these points in a text.

It is expected that readers in this study will differ in their success and in their application of global processing strategies. A less successful reader will presumably apply a strategy that does 
not differentiate between topic sentences and paragraph sentences and will not invest additional time and effort in processing the headings and topic shifts. According to Lorch, et al. (1987), such readers will not pay additional attention to headings or topic shift sentences, will not additionally process paragraph endings or beginnings, or section breaks, and they will not backtrack to important information. While rereading or backtracking to important points in a text is applied by more and less competent readers, the difference is that successful readers pay attention to significant text parts like headings or topic sentences, while less successful readers do not.

\section{Reading Strategy Identification with Eye Tracking}

For the purpose of this study, it was assumed that in a reading comprehension task involving an expository text the readers would apply one of four possible strategies, one being a selective strategy and the other three nonselective (see Hyöna et al., 2002). If a selective strategy is used, the reader will apply additional cognitive effort and time when reading a sentence with the introduction of a new topic, and this will be the case in the subsequent reading as well. If a nonselective strategy is used, the reader will not give specific attention to topical parts of the text. Some nonselective readers will use the repeated reading strategy and still remain nonselective, and some will not reread. Selective processing as a reading strategy has up to now been supported by Hyöna (1994), Hyöna et al., (2002) and Lorch, et al. (1985, 1987).

In this study, the Eyelink eye tracking device (SR Research Ltd.) was used, with the aim of ascertaining which global processing strategies were applied by competent L2 speakers when reading a text in L2 on a computer screen. An inductive approach was applied in order to ascertain various possible global processing strategies used by the readers in the study. Previous research has dealt with eye tracking methodology in reading tasks, but rarely with the identification of processing strategies. A specific advantage of this study is that no reading strategies were defined beforehand, which allowed for variations and the potential identification of new strategies applied by users (cf. Hyöna et al., 2002).

Rayner (1998) used eye-tracking to identify several issues in reading tasks, while Olson et al. (1985), investigated local reading strategies. Global reading processing strategy was investigated by previous researchers (Blanchard and Iran-Nejad, 1987; Hyöna, 1995; Shebilske and Fisher, 1983; Vauras et al., 1992), while Hyöna et al. (2002) investigated the differences in online reading strategies for global text processing.

Specific advantages of online text processing are that it is possible to collect more than one processing element simultaneously while maintaining a high resolution of temporal and spatial data (Hyöna et al., 2002). When reading a text on the screen, the reader is not forced to read in a linear fashion: He or she can read text items in any order they choose. The technique of online text processing enables researchers to get a complete overview of a person's reading strategies used during the task. The data obtained from each reader was analysed via clusters, and 
subsequently similar profiles were categorised based on dependent variables. In order to avoid obtaining chance patterns when analysing the data, the study was based on two texts rather than only one for each reader. In this way it was possible to check the reliability of the clustering technique applied. Finally, it was checked whether higher grades in the English language university course correlated with a more successful reading strategy among students.

\section{Overview of the Experiment}

In this experiment, eye-fixation data was recorded for university students whose competency in the English as a foreign language is at $\mathrm{C} 1$ level of the Common European Reference Framework for Languages. Language skills were ascertained from the grades in the English Language Skills Course obtained by the students in the previous year. Students are graded on a scale from 1 (fail) to 5 (excellent), and all the students passed the English language course from the previous year.

The assignment involved reading two expository texts in the English language containing multiple topics. The frequency and duration of eye fixations were recorded for each text sentence. Fixations were categorised as forward fixations, re-inspections, look-backs and lookfroms (see Hyöna et al., 2002). In forward fixations, the reader's eyes move toward a previously unread part of a given sentence, indicating the first reading of information in a text. Reinspections refer to going back to a part of the current sentence and point to integration of information and reflection upon it. Look-backs refer to eye movements back to a part of text beyond the current sentence, which is indicative of global reflection or integration of information. Look-forwards indicate eye movements forward in text beyond the current sentence.

Text structure items in this analysis were headings $(\mathrm{H})$, beginning (Topic 1, Topic 2) and ending sentence (End 1, End 2) of each paragraph on a page (two paragraphs per page) and remaining sentences (Other). Sentences at the end of paragraph 2 in a text were also end-ofsection sentences, which carried additional weight in the overall integration of the text.

\section{Empirical Study}

\section{Participants}

Participants included in this study were 43 university students: 19 third year students at the Faculty of Political Science and 24 third year students at the Faculty of Economy at the University of Zagreb. Consent was obtained from each participant in the study and the data was anonymised for each participant. An overall number of 43 students studying an English language course as a part of their study curriculum were included in the study, and their reading strategies were analysed for two expository texts in English as the target language. The English 
language courses were designed for students of the second, third and fourth year of study at C1 level of the Common European Reference Framework for Languages and the texts were prepared for that level of L2 study. Of the 43 participants, 24 were female and 19 male, in the age range of 18 to 27 years.

\section{Methodology}

Eye-tracking data was collected via the SR Research EyeLink 1000 eye tracker, which measures eye movements by detecting the position of the pupil in a video image. The system works by emitting infrared light onto the eyes of the participant and recording the image reflected from each eye. For this purpose, a high-resolution infrared camera was applied. The camera and light source were placed beneath the experiment monitor on which the texts were displayed. During the experiment, pupil movements and reflections of the corneum were measured. Pupil movements (vertical and horizontal) indicated the participant's attention on sections of the text, and corneal reflection pointed to head movements, which helped to distinguish between actual pupil movements.

\section{Texts}

Two English language texts were used in this study and were presented online on a computer screen. One was an expository text on GMO, and another on the new German law regulating social media. Both texts were 1500 words long.

The GMO text began with a summary and was organised into four sections with four headings. The first section concerned GMO toxicity and contained concerns and scientific research as topics. The section discussed anti-GMO advocacy, GMO-safety research and research examples. Section two dealt with the effect of GMO over time. It discussed the health concerns related to fertility and the build-up of toxicity over time. The third section dealt with a potential gene mutation caused by GMO, and discussed two concerns: mutagenicity and the possibility of transferring the modified DNA to the DNA of the person who eats it. Studies related to these concerns were discussed along with their results. Section four presented the conclusion, which ended with an argument in favour of GMO safety. The text is organised according to topics, which were structured with headings in bold. Each topic in a section was discussed in two paragraphs containing information on different aspects. In each section the first sentence offered information on the section topic and on the topic of the paragraph. The first sentence of each paragraph introduced the paragraph topic. For the purpose of the study task, the text was graphically prepared so that each topic was discussed on a separate page.

The second text was on the new German law regulating social media. The text starts with an introduction and announces the four topics it discusses. The four topics of the text deal with misconceptions about the new law. Each section of the text is numbered and starts with a 
heading written in bold. Each section consists of two or more paragraphs. Section one discusses the misconception of the new law targeting hate speech. Section two discusses the misconception on users being fined if they fail to remove certain content. Section three discusses the misconception on companies having 24 hours to remove illegal content, and section four discusses the question whether the new law will have an adverse effect on freedom of speech.

Both texts were presented on the computer, in Arial font sized 12, double-spaced, with a maximum of twelve (12) lines of text for each page. The order in which the participants were shown the texts was changed for each participant; half of all the students read the GMO text first and the social media text second, and the other half read both texts in the reversed order.

\section{Procedure}

The preparations for the experiment consisted of checking and adjusting the equipment for each participant. For each participant, the eye tracking device was calibrated before the actual task. Each participant was instructed to read the text in order to summarise it after reading. Before the actual experiment, each reader was presented with a short trial text, to help them familiarise themselves with the equipment and understand the instructions provided. There were no time restrictions in the experiment and participants were allowed to read as slowly and carefully as they needed, with the limitation that it was not possible to return to the previous page once they moved forward to the next page.

After each participant read both texts, they were presented with a reading-span test (see Daneman and Carpenter, 1980). In this test, each participant was asked to read aloud several sets of sentences which were topically unrelated (see Hyöna et al., 2002). Each sentence was written on a card with a white background and presented to the reader by the experimenter, one by one, for a specific set. The task required the participant to memorise and repeat the last word in each sentence in a given set of sentences. The sets were gradually increased, with each set size being repeated three times before moving on to a larger set, whereupon they were increased by one sentence at a time. The participant was presented with sentence sets as long as he or she was able to recall the ultimate words of all the sentences in a given set. Each participant was scored according to the maximum number of words they were able to recall for a given set. Each test lasted approximately 15 minutes. Thereafter the participants were asked to summarise the first text they read. The whole experiment lasted around 1.5 hours.

\section{Data Collection}

For each sentence in both texts, eye movements were measured according to duration and frequency of four kinds of eye movement: forward fixations, re-inspective fixations, look- 
backs and look-froms. In this way, eight different parameters were obtained, four kinds of data for the reading of two texts.

Forward fixations denote eye movements from left to right, while re-inspective fixations, lookbacks and look-froms signify eye movements from right to left. Forward fixations and reinspective fixations are used during the first reading of a given sentence and denote eye movement on a new part of text, while re-inspections stand for eye movement when rereading parts of the currently read sentence. Look-backs are related to eye movement directed back from the currently read sentence onto a previously read sentence, denoting rereading of a previously read part of text. Look-froms are eye movements directed away from the currently read sentence back to a sentence that has already been read.

In previous literature eye fixations have been categorised as progressive and regressive (Rayner, 1998), but in this study we chose to differentiate between four types of fixations, as used by Hyöna et al. (2002:47). The reason being that re-inspective fixations and look-backs are not always regressive, even though they are related to eye movement from right to left. Specifically, every re-inspection and look-back will start as a regressive eye movement, but subsequently they can also be progressive.

In the first reading of each sentence, the frequency and duration of forward fixation (1) and reinspective fixation (2) were measured. Frequency and duration were also measured for lookbacks (3), which refer to looking back over one and the same sentence that has already been read once, and look-froms (4), which refer to looking from one sentence back to another that has already been read. Due to various sentence lengths, ratio per character was computed in order to balance out the differences. The first two aspects, forward fixation and re-inspection, are attributed to the cognitive effort involved in processing a sentence at first reading. Look backs and look froms are attributed to tasks of resolving difficulties in comprehension and integrating pieces of information.

Upon measuring calculation for individual sentences, an average was computed for sentences with mutual functions in relation to the structure of the text. In this study six types of sentences were identified. Mean values were calculated for the headings in the text, sentences at the beginning and end of paragraph 1 and paragraph 2 of each page, and for all the remaining sentences. An overall of 48 data points were computed in each text for each individual participant. For the three aspects mentioned previously there are two dependent variables, frequency and duration, and six sentence types: heading, topic sentence in paragraph 1, end sentence in paragraph 1 , topic sentence in paragraph 2 , end sentence in paragraph 2 and remaining sentences. 


\section{Clustering Technique for Reading Strategy Categorisation}

Participant reading strategies were ascertained by grouping eye-tracking information into clusters. In line with previous studies of this kind (see Hyöna, 1995, Hyöna et al., 2002), there was a high degree of correlation for eye-movement frequency and eye-movement duration measures. This made it possible to use only one set of data - for duration or frequency - as both sets create redundancy. However, by using both sets, more certainty is achieved when reaching the final conclusion. In this study we used both sets of data but concentrated on eye fixation duration. Cluster analysis demands a conversion of scores into standard z-scores for the purpose of equal weighting in the data analysis. A hierarchy based, agglomerative procedure of clustering (Ward's method) was used in the process. According to previous studies, this is a very powerful and popular technique, and aims to minimise the sum of square for incidences in any two given clusters created (see Morey et al., 1983).

Data from 43 participants was used for two texts, which resulted in 86 cases in the final data set. The data obtained in the study was organised into clusters, which identify individual differences in participants' global text reading strategies. The appropriateness of the cluster approach for this measurement can be checked by analysing if an individual reader applied the same reading strategy to both texts read. To confirm this, the proportion of readers applying the same strategy for both texts was calculated. The resulting proportion amounted to .88: after correction for chance it amounted to .82. Clusters that were significantly different from each other confirmed consistency of data. The overall result confirms that cluster analysis is an appropriate method for ascertaining individual reading strategies in this experiment.

Each cluster was compared to other clusters at sentence level by applying independent group tests. Individual sentence types, "heading", "topic" and "end" were compared against sentences marked as "other" within each cluster. In addition, for re-inspective fixations and look backs, a nonparametric Mann-Whitney U test was applied, as well as a Friedman test. Results showed statistically significant differences above 0.05 .

Data analysis led to the conclusion that the optimal solution was a categorisation into four clusters. A fifth cluster option was not optimal, as such a cluster did not show enough differentiation in comparison to the first four reading strategy clusters.

\section{Global Text Processing Strategies in Foreign Language Reading}

In this study we identified four individual clusters and global L2 text processing strategies, as shown in Figures 1, 3, 5 and 7, for the eye fixation duration, and in 2, 4, 6 and 8 for fixation frequency. 
Each strategy profile was created based on a statistical analysis of means for the two examined texts. Figures with odd numbers show the duration of eye movement fixations for each cluster of readers, and figures with even numbers show the frequency of fixations for each respective cluster of readers. The data shown is recorded for different types of sentences (heading, topic, end, other).

Partly Selective Fast Linear Readers

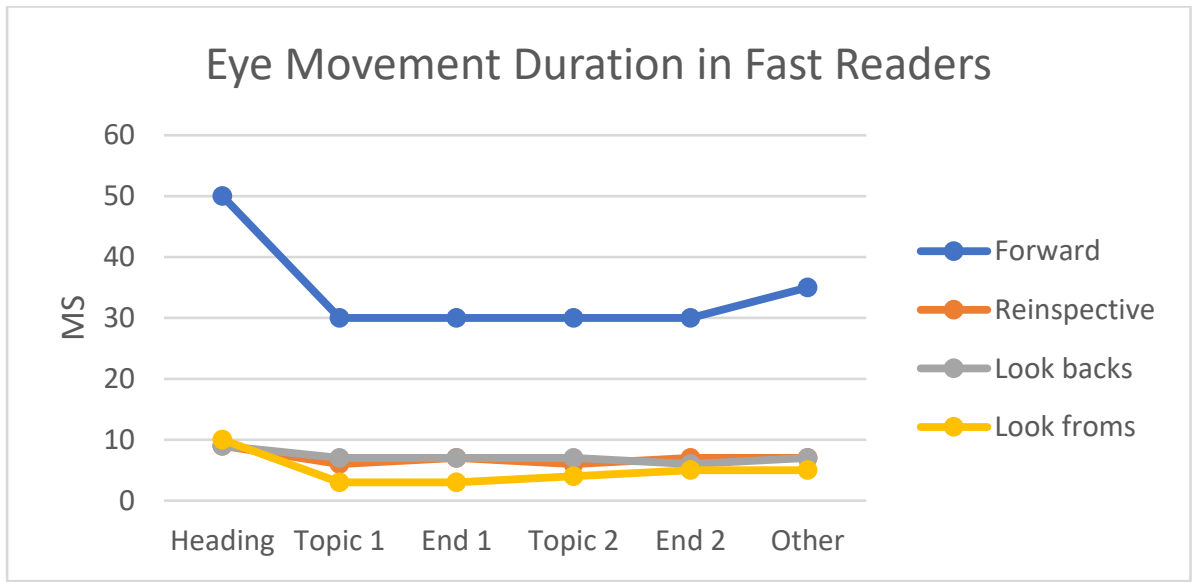

Figure 1. Duration in milliseconds per character for eye movement in partly selective fast linear readers: forward fixations, re-inspective fixations, look-backs and look-froms $(\mathrm{N}=15)$

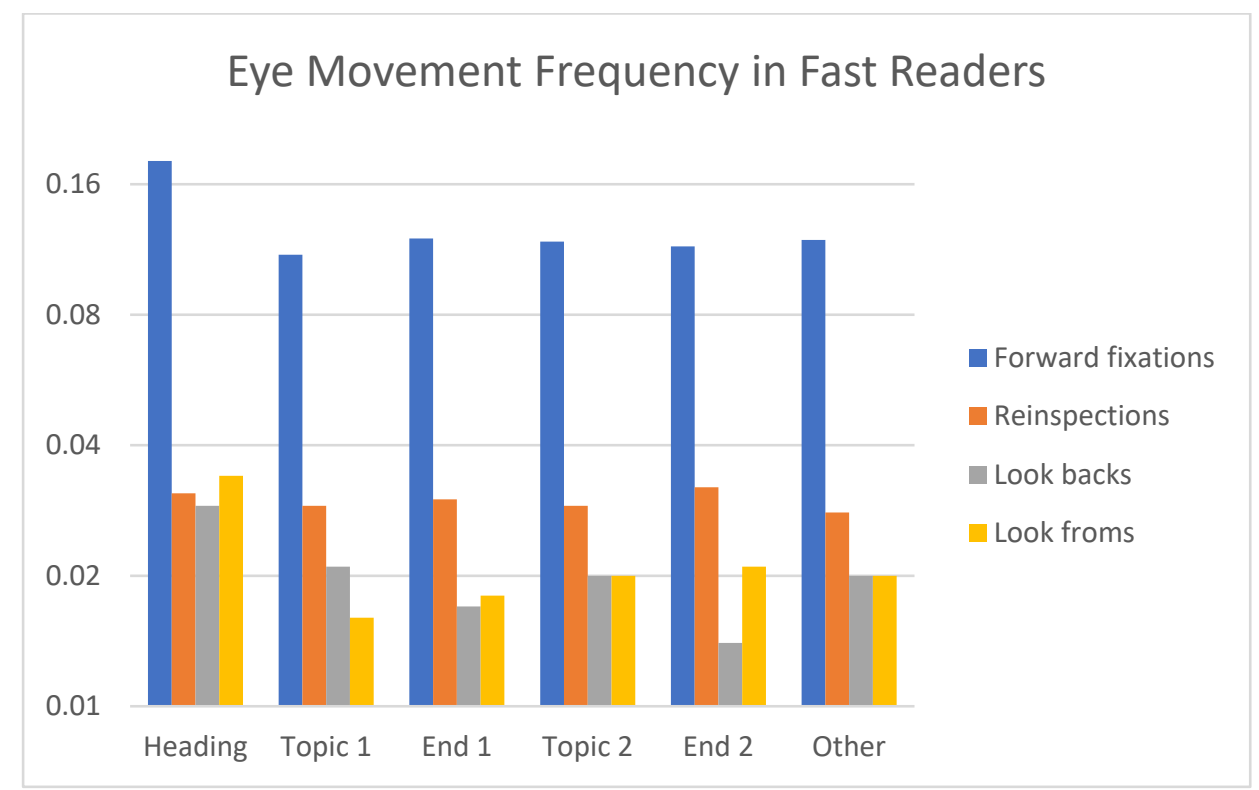

Figure 2. Frequency of eye movements in partly selective fast linear readers: forward fixations, re-inspections, look-backs and look-froms $(\mathrm{N}=15)$

An overall number of 15 participants were identified as partly selective fast linear readers, who dominantly used forward fixations as a strategy in both texts (blue line). This group is significantly different from other readers regarding each sentence type apart from headings. In 
this cluster readers used the most time for forward eye fixations and the smallest amount of time for re-inspections, look backs and look froms. The reading strategy applied by these readers is linear, as they did not use time to process or reprocess information and to re-inspect. Their attention when looking back was mostly given to headings. When re-inspecting, this type of readers usually focused on paragraph 2 end sentences.

\section{Partly Selective Slow Linear Readers}

In this cluster there were 16 readers, who did not look back often and did not differentiate a lot between different sentence types when looking back, as their eyes stay fixed for a similar duration on topic and end sentences, as well as other sentence types. In that sense they are similar to fast readers, but they differ in the time they need to process information. When looking at forward fixation duration, this type of reader showed the longest time spent, in comparison to other readers. These readers, along with nonselective readers, had significantly lower grades in the English language skills course in comparison to fast and structural readers.

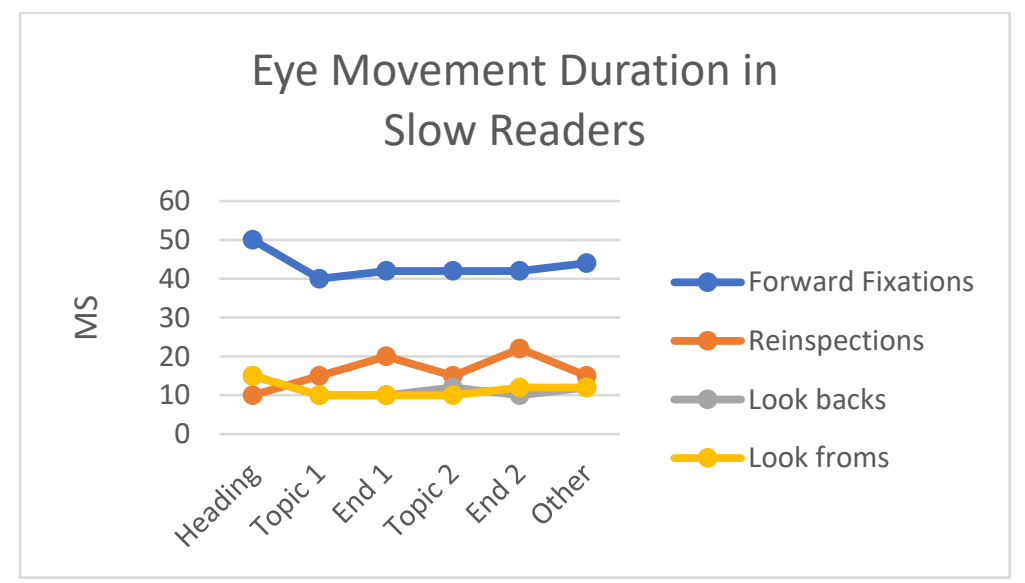

Figure 3. Duration in milliseconds per character for eye movement in partly selective slow linear readers: forward fixations, re-inspective fixations, look-backs and look-froms $(\mathrm{N}=16)$ 
International Journal of Innovation, Creativity and Change. www.ijicc.net

Volume 15, Issue 5, 2021

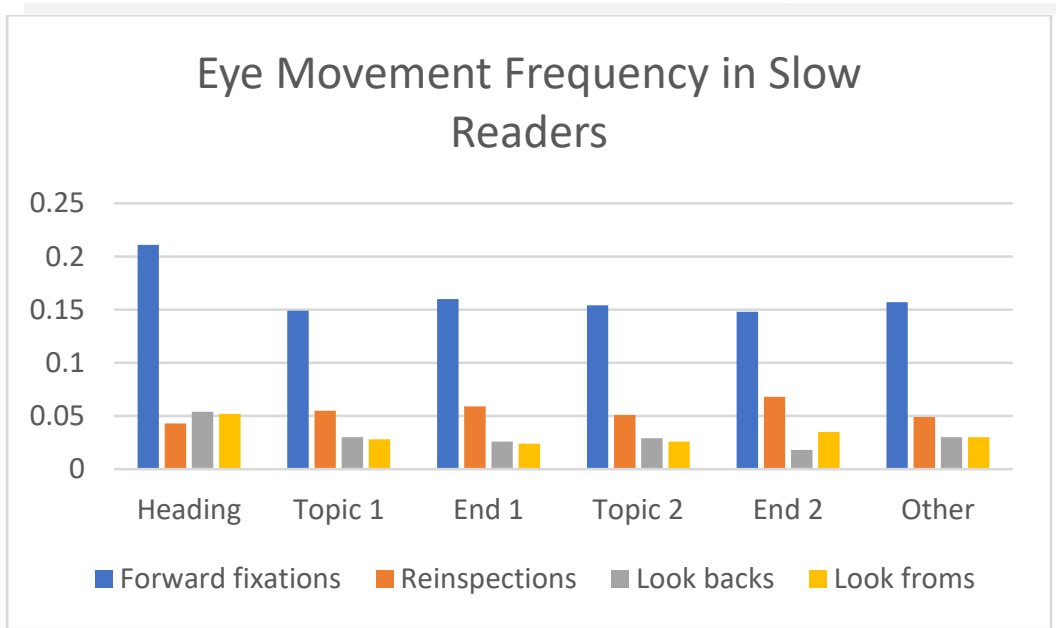

Figure 4. Frequency of eye movements in partly selective slow linear readers: forward fixations, re-inspections, look-backs and look-froms $(\mathrm{N}=16)$

\section{Selective Structural Readers}

The third identified cluster in this study are selective structural readers, who focus on the topic structure when processing the text. An overall of seven (7) students were identified in this cluster. These readers took a longer time to process headings, as can be seen in the forward fixations (blue line) and in look-backs (grey line), but also in re-inspective fixations (red line). In comparison to partly selective fast and slow linear readers, this group spends much more time on headings. Within this cluster it is noted that the readers spend more time fixing their eyes on headings and end sentences in the last paragraph. These readers recognise and pay attention to structural segments of the texts, as they looked back from end sentences more often than other readers. They looked back from End 2 sentences more frequently than from other sentences and their re-inspective fixations were longer on end sentences in comparison to other sentences.

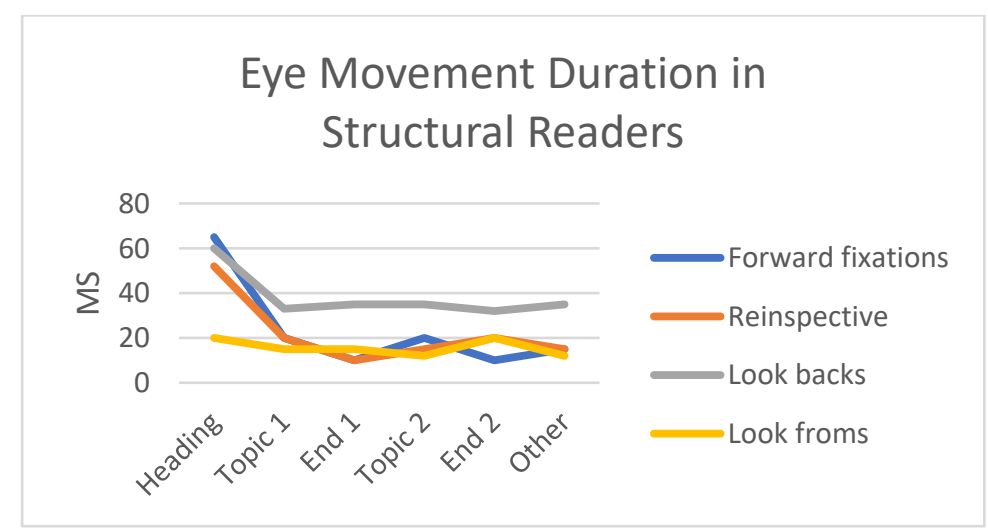

Figure 5. Duration in milliseconds per character for eye movement in selective structural readers: forward fixations, re-inspective fixations, look-backs and look-froms $(\mathrm{N}=7)$ 
International Journal of Innovation, Creativity and Change. www.ijicc.net

Volume 15, Issue 5, 2021

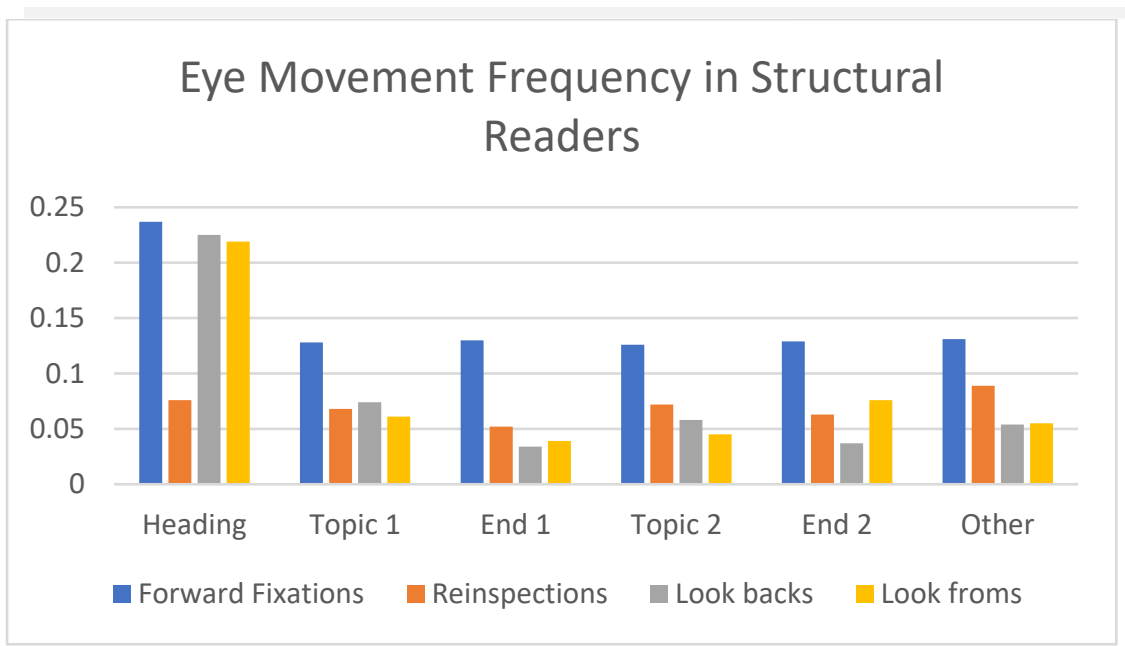

Figure 6. Frequency of eye movements in selective structural readers: forward fixations, reinspections, look-backs and look-froms $(\mathrm{N}=7)$

\section{Nonselective Readers}

In this cluster we could identify an overall number of five (5) participants, who did not appear to have a strategy in text processing. These readers tend to reread the text more than other participants. They do this by looking back more frequently to previously read sentences, but in doing so they do not differentiate between topic and other sentences. These readers look back longer and more often than other three clusters of readers.

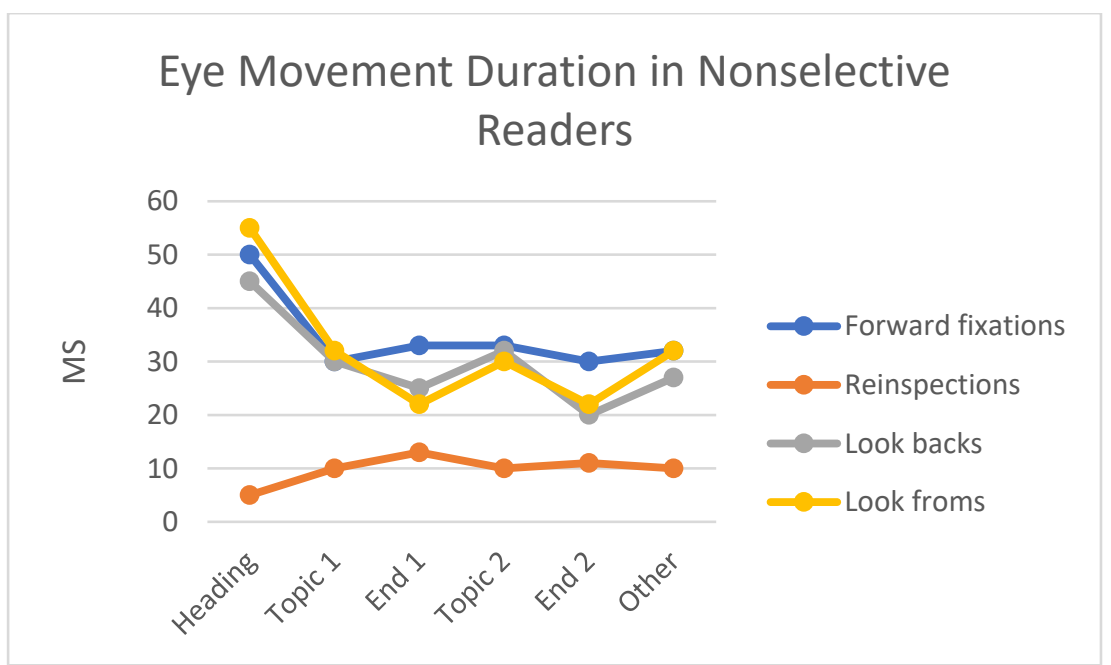

Figure 7. Duration in milliseconds per character for eye movement in nonselective readers: forward fixations, re-inspective fixations, look-backs and look-froms $(\mathrm{N}=5)$ 


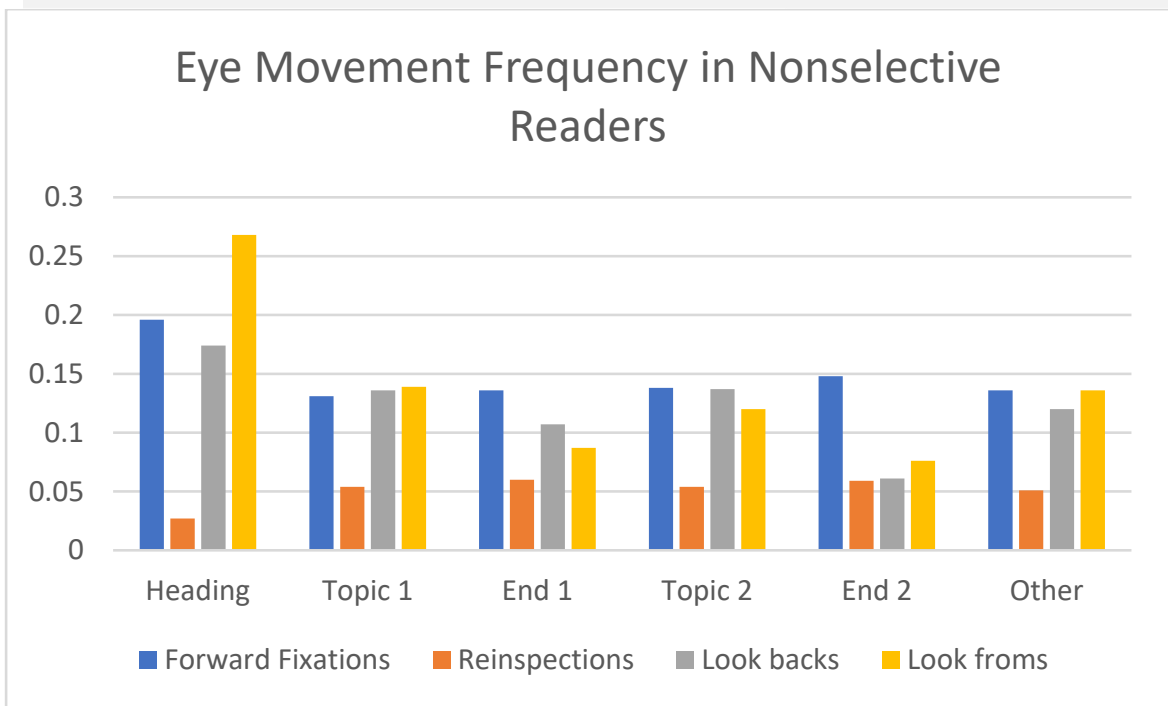

Figure 8. Frequency of eye movements in nonselective readers: forward fixations, reinspections, look-backs and look-froms $(\mathrm{N}=5)$

In the experiment the readers were also administered a reading span test (see Daneman and Carpenter, 1980), in order to ascertain the differences in the span of their working memory. According to results from one-way ANOVA, differences were significant between clusters of readers; F (2, 33), MSE.82.

\section{Discussion}

In this study it was analysed which reading strategies are applied by university students who are English language speakers at $\mathrm{C} 1$ level. Results show that there are significant differences in the strategies readers use when processing an expository text in L2.

Figures 1, 3, 5 and 7 show that all four clusters of readers give more attention to headings when reading the text, which indicates that all readers use some type of selective strategy. However, as the heading is visually separated from the rest of the text, this can also have contributed to longer eye fixations on that part of the text.

Four separate reader clusters with three different global processing strategies were identified, based on the patterns of re-inspection and lookbacks. Re-inspection is noted with partly selective slow linear readers and is very weakly pronounced with fast readers. As these readers also rarely look back, their reading rate is much faster in comparison to other readers. However, partly selective fast linear readers in our study are not as fast as those in the same category in previous studies (cf. Hyöna et al. 2002). This is probably due to reader proficiency in the second language, as students are at $\mathrm{C} 1$ level of English. Even though partly selective slow linear readers also tend to rarely look back, they differ from fast readers in the frequency and duration of such fixations. Partly selective fast linear readers demonstrated very little re-inspection and 
look-backs, but proved to be very competent in summarising the texts. Also, this cluster of readers showed high grades in their English language course at university.

Partly selective slow linear readers demonstrated little selection in reading and a large amount of re-inspection during their first reading of a given text. This cluster of readers had lower grades in their English language course and also had problems when summarising the text in this experiment. In addition, they demonstrated the lowest working memory capacity in comparison to other clusters. It can be concluded that this cluster of readers has difficulties when processing a text globally, as they have difficulty in comprehension and sentence processing.

Selective structural readers are unique in the sense that these readers are selective in their text processing methods as opposed to the first two clusters. They are high performers, as they have the highest grades in the English language at university and were the most successful in the text summarisation task. During the reading task, they concentrated more on the headings and paragraph end sentences. Also, they demonstrated larger text reading spans than other readers. When comparing our findings to previous research results, there are correspondences in the conclusions that a high level of sensitivity to topical sentences indicates a high competence in text summarisation (see Lorch, R.F., et al., 1987; Hyöna et al., 2002). In this study it could be observed how competent readers who apply the method of topic structure proceed when reading a text. Results have shown that sentences at the end of each section are key points for the processing and structuring of previously acquired information.

It was also observed that university students differ in the strategies they use in reading comprehension and that not all competent and successful students use the topic structure method. Actually, the most widely used strategy was that of fast linear processing, which demonstrated very little sensitivity to topic sentences. This points to a need for training of students in order to improve their topic processing skills in text reading. 
International Journal of Innovation, Creativity and Change. www.ijicc.net

Volume 15, Issue 5, 2021

\section{REFERENCES}

Blanchard, H. E., Iran-Nejad, A. (1987). Comprehension processes and eye movement patterns in the reading of surprise-ending stories. Discourse Processes, 10: 127-138.

Daneman, M., Carpenter, P. A. (1980). Individual differences in working memory and reading. Journal of Verbal Learning and Verbal Behavior, 19: 450-466.

Fletcher, C. R.,, Bloom, C. P. (1988). Causal reasoning in the compre- hension of simple narrative texts. Journal of Memory and Language, 27: 235-244.

Goldman, S. R., Saul, E. U. (1990). Flexibility in text processing: A strategy competition model. Learning and Individual Differences, 2: 181-219.

Graesser, A. C., Bertus, E. L.,, Magliano, J. P. (1995). Inference generation during the comprehension of narrative text. In R. F. Lorch \& E. J. O'Brien (Eds.), Sources of coherence in reading (pp. 295-320). Hillsdale, NJ: Erlbaum.

Haberlandt, K.,, Graesser, A. C. (1989). Processing of new arguments at clause boundaries. Memory \& Cognition, 17: 186-193.

Hyöna, J. (1994). Processing of topic shifts by adults and children. Reading Research Quarterly, 29: 76-90.

Hyöna, J. (1995). An eye movement analysis of topic-shift effect during repeated reading. Journal of Experimental Psychology: Learning, Memory, and Cognition, 21: 1365-1373.

Kieras, D. E. (1981). Component processes in the comprehension of simple prose. Journal of Verbal Learning and Verbal Behavior, 20: 1-23.

Kintsch, W.,, van Dijk, T. A. (1978). Toward a model of text comprehension and production. Psychological Review, 85: 363-394.

Lorch, E. P.., Lorch, R. F., Jr., Gretter, M. L., Horn, D. G. (1987). On-line processing of topic structure by children and adults. Journal of Experimental Child Psychology, 43: 81-95.

Lorch, R. F., Jr., Chen, A. H. (1986). Effects of number signal on reading and recall. Journal of Educational Psychology, 78: 263-270.

Lorch, R. F., Jr., \& Lorch E. P. (1996). Effects of organizational signals on free recall of expository text. Journal of Educational Psychology, 88: 38-48. 
Lorch, R. F., Jr., Lorch E. P., Matthews, P. D. (1985). On-line processing of the topic structure of a text. Journal of Memory and Language, 24: 350 -362.

Lorch, R. F., Jr., Lorch E. P.,, Morgan, A. M. (1987). Task effects and individual differences in on-line processing of the topic structure of a text. Discourse Processes, 10: 63-80.

McKoon, G.,, Ratcliff, R. (1992). Inference during reading. Psychological Review, 99: 440466.

Morey, L. C.,, Blashfield, R. K.,, Harvey, A. A. (1983). A comparison of cluster analysis techniques within a sequential validation framework. Multivariate Behavioral Research, 18: 309-329.

Norris, M.L. (2015): Will GMOs Hurt My Body? The Public's Concerns and How Scientists Have Addressed Them. http://sitn.hms.harvard.edu/flash/2015/will-gmos-hurt-mybody/ (Last accessed on December 20th, 2020).

Olson, R. K., Kliegl, R., Davidson, B. J., Foltz, G. (1985). Individual and developmental differences in reading disability. In G. E. MacKinnon \& T. G. Waller (Eds.), Reading research: Advances in theory and practice (Vol. 4, pp. 1-64). New York: Academic Press.

Pressley, M.,, Afflerbach, P. (1995). Verbal protocols of reading: The nature of constructively responsive reading. Hillsdale, NJ: Erlbaum.

Rayner, K. (1998). Eye movements in reading and information processing: 20 years of research. Psychological Bulletin, 124, 372-422.

Shebilske, W. L.,, Fisher, D. F. (1983). Eye movements and context effects during reading of extended discourse. In K. Rayner (Ed.), Eye movements in reading: Perceptual and language processes (pp. 153-179). New York: Academic Press.

Theil, S. (2018): Germany's Legal Crackdown on Social Media: Four Misconceptions Dispelled. https://theconversation.com/germanys-legal-crackdown-on-social-mediafour-misconceptions-dispelled-96311 (Last accessed on December 20th, 2020).

Vauras, M., Hyöna, J., Niemi, P. (1992). Comprehending coherent and incoherent texts: Evidence from eye movement patterns and recall performance. Journal of Research in Reading, 15: 39-54. 